\title{
Overexpression of gelsolin reduces the proliferation and invasion of colon carcinoma cells
}

\author{
WEN-XIANG LI*, MENG-XUAN YANG*, XIN-QIANG HONG, TIAN-GENG DONG, \\ TUO YI, SHENG-LI LIN, XIN-YU QIN and WEI-XIN NIU
}

Department of General Surgery, Zhongshan Hospital of Fudan University, Shanghai 200032, P.R. China

Received March 6, 2015; Accepted January 4, 2016

DOI: $10.3892 / \mathrm{mmr} .2016 .5652$

\begin{abstract}
The enhanced motility of cancer cells via the remodeling of the actin cytoskeleton is crucial in the process of cancer cell invasion and metastasis. It was previously demonstrated that gelsolin (GSN) may be involved as a tumor or a metastasis suppressor, depending on the cell lines and model systems used. In the present study, the effect of GSN on the growth and invasion of human colon carcinoma (CC) cells was investigated using reverse transcription quantitative polymerase chain reaction and western blotting. It was observed that upregulation of the expression of GSN in human CC cells significantly reduced the invasiveness of these cells. The expression levels of GSN were observed to be reduced in $\mathrm{CC}$ cells, and the reduced expression level of GSN was often associated with a poorer metastasis-free survival rate in patients with $\mathrm{CC}(\mathrm{P}=0.04)$. In addition, the overexpression of GSN inhibited the invasion of CC cells in vitro. Furthermore, GSN was observed to inhibit signal transducer and activator of transcription (STAT) 3 signaling in CC cells. Together, these results suggested that GSN is critical in regulating cytoskeletal events and inhibits the invasive and/or metastatic potential of CC cells. The results obtained in the present study may improve understanding of the functional and mechanistic links between GSN as a possible tumor suppressor and the STAT3 signaling pathway, with respect to the aggressive nature of $\mathrm{CC}$. In addition, the present study demonstrated the importance of GSN in regulating the invasion and metastasis of CC cells at the molecular level, suggesting that GSN may be a potential predictor of prognosis and treatment success in CC.
\end{abstract}

Correspondence to: Professor Wei-Xin Niu, Department of General Surgery, Zhongshan Hospital of Fudan University, 180 Fenglin Road, Shanghai 200032, P.R. China

E-mail: niuwx021@126.com

${ }^{*}$ Contributed equally

Key words: overexpression, gelsolin, colon carcinoma, signal transducer and activator of transcription 3, tumor metastasis, prognostic factor

\section{Introduction}

Colon carcinoma (CC) is the second most common type of malignant tumor and ranks as the third highest cause of cancer-associated mortality worldwide (1-3). Although early $\mathrm{CC}$ detection and treatment can lead to a good prognosis, the survival rate is low when metastasis occurs (4-6). Due to the numerous contributing factors in the development of $\mathrm{CC}$, the pathogenesis remains unclear, therefore, the investigation of novel therapeutic strategies is a key focus in CC research.

Investigations of $\mathrm{CC}$ have focused on the identification of dysregulated genes, protein markers, non-encoding RNA, including miroRNA-145 (7) and additional prognostic molecular markers. The aim of these investigation has been to formulate novel strategies for the treatment of $\mathrm{CC}$ on the basis of identifying abnormal genes, key molecular targets and CC-associated signaling pathways (8).

It is widely known that the metastasis of a malignant tumor from the primary source to other tissues and organs is a serious complication in cancer, and is key in the treatment of malignant tumors $(9,10)$. Cancer cells acquire motility through the remodeling of the actin cytoskeleton, which is a key process involved in the invasion and metastasis of cancer cells (11).

Gelsolin (GSN) is a widely expressed actin regulator, which is important in regulating cell motility by severing actin $(12,13)$. In addition, GSN is able to regulate cell morphology, proliferation and apoptosis (14). A previous study demonstrated that the expression levels of GSN are reduced in breast, urinary bladder, colon, kidney, ovary, prostate, gastric and urinary system cancer (15).

However, whether there is a direct association between the expression of GSN and tumor development remains to be fully elucidated (16-18). Previous studies have reported that the overexpression of GSN promotes the motility of tumor cells and enhances their invasiveness by regulating various signaling pathways, including the phosphoinositide 3-kinase (PI3K) and Ras-PI3K-Rac pathways $(19,20)$. However, it has been demonstrated that GSN can inhibit epithelial-mesenchymal cell transformation in breast cancer (15), and act as a suppressor of metastasis in B16 melanoma cells (21).

In the present study, the role of GSN in the proliferation and invasion of human CC cells was investigated in order to determine whether the overexpression of GSN attenuates the invasiveness of these cells, and whether a reduction in 
the expression of GSN is associated with the invasiveness of human $\mathrm{CC}$ cells or the prognosis of CC patients. This may determine whether stabilizing the expression of GSN inhibits the invasiveness of CC cells.

The signal transducer and activator of transcription (STAT) protein family regulates the expression of several genes, which are involved in cell survival, proliferation and apoptosis (22-25). STAT3 is associated with tumor occurrence via promotion of the proliferation and invasion of several types of cancer cell, including human CC cells (26-29). The inhibition of STAT3 was observed to inhibit the proliferation of human CC cells, indicating that STAT3 may be a potential target in the treatment of CC (30-34).

To further elucidate the role of GSN in CC cells, the present study investigated the effect of the expression of GSN on the STAT signaling pathway, to determine how GSN coordinates with STAT3 to regulate metastasis in CC.

\section{Materials and methods}

Tissue specimens and cell culture. A total of 30 paired primary colon tumors and corresponding normal colon tissue specimens were obtained from patients with CC (gender, 13 men and 17 women; mean age, 64.43 years; age range, 23-93 years) who were admitted to Zhongshan Hospital of Fudan University (Shanghai, China) between 2009 and 2011, and from whom informed written consent was obtained.

The selection of CC cases was based on a clear pathological diagnosis and follow-up data in patients who had not previously received local or systemic treatment. Tumor stages were defined, according to the 2002 American Joint Committee on Cancer/International Union against Cancer tumor-node-metastasis classification system (35). The present study was approved by the Institutional Research Ethics Committee of Zhongshan Hospital of Fudan University. The SW480 and HT29 CC cell lines were cultured in RRMI medium (Gibco; Thermo Fisher Scientific, Inc., Waltham, MA, USA) with $10 \%$ fetal bovine serum (FBS; Gibco; Thermo Fisher Scientific, Inc.). The SW620 and HCT116 CC, and the normal CCD-18Co colon cell lines were maintained in Dulbecco's modified Eagle's medium (DMEM; Gibco; Thermo Fisher Scientific, Inc.) supplemented with 10\% FBS. All cell lines were purchased from the Shanghai Cell Institute Country Cell Bank. (Shanghai, China). During tumor resection surgery, fresh tissue samples were harvested from the recruited patients; tumor tissues were obtained from the center of the tumor and adjacent normal tissues from $5 \mathrm{~cm}$ away from the tumor margin. The tissue samples were snap-frozen and preserved at $-80^{\circ} \mathrm{C}$.

RNA isolation and reverse transcription-quantitative polymerase chain reaction ( $R T-q P C R)$. Total RNA was extracted using TRIzol reagent (Invitrogen; Thermo Fisher Scientific, Inc.). cDNA was synthesized using a PrimeScript RT Reagent kit (Promega Corporation, Madison, WI, USA). The expression level of GSN was analyzed relative to the level of the $\beta$-actin gene transcript using an Applied Biosystems 7300 PCR system (Thermo Fisher Scientific, Inc.). First-strand cDNA $(2 \mu 1)$ was amplified in a $20 \mu \mathrm{l}$ PCR reaction mixture, containing $10 \mu 1$ 2X SYBR green PCR master mix (Qiagen, Hilden,
Germany), $0.4 \mu 1$ 50X ROX Reference Dye (Thermo Fisher Scientific, Inc.), $0.4 \mu \mathrm{l}$ of each specific primer set and $\mathrm{ddH}_{2} \mathrm{O}$ added to a total volume of $20 \mu \mathrm{l}$. The primer sequences were as follows: $\beta$-actin, forward 5'-AGCGAGCATCCCCCAAAG TT-3' and reverse 5'-GGGCACGAAGGCTCATCATT-3'; and GSN, forward 5'-GGTGTGGCATCAGGATTCAAG-3' and reverse 5'-TTTCATACCGATTGCTGTTGGA-3'. The primer sequences were purchased from (Invitrogen; Thermo Fisher Scientific, Inc.). The amplification was performed under the following conditions: $10 \mathrm{~min}$ at $95^{\circ} \mathrm{C}$ for one cycle, 40 cycles of $95^{\circ} \mathrm{C}$ for $15 \mathrm{sec}$ and $60^{\circ} \mathrm{C}$ for $60 \mathrm{sec}$. The RT-qPCR data were quantified using the comparative $\mathrm{Cq}$ method (36).

Construction of the pGEM-T-GSN vector. GSN target fragments (Wegene) were recovered and purified using $1 \%$ low melting agarose gel electrophoresis (Sigma-Aldrich, St. Louis, MO, USA) and a DNA purification kit (K0512; Thermo Fisher Scientific, Inc.). The purified gene fragments and pGEM-T vector (Promega Corporation, Madison, WI, USA) were combined according to the manufacturer's instructions, and transformed into JM101 competent cells (prepared by the calcium chloride method). Single colonies were randomly selected and added to lysogeny broth (InvivoGen, San Diego, CA, USA) liquid medium (containing ampicillin; Sigma-Aldrich) at $37^{\circ} \mathrm{C}$ and agitated for $12 \mathrm{~h}$. Following plasmid extraction, the products were identified by restriction enzyme digestion using HindIII and KpanI (New England BioLabs, Inc., Ipswich, MA, USA). The positive plasmids of the double digested results were sent to Shanghai Invitrogen Biotechnology Co., Ltd. (Shanghai, China) for forward and reverse sequencing. The correct plasmid was identified from the sequencing results and termed pGEM-T-GSN.

DNA transfection. Cells were seeded uniformly into 6-well plates at a density of $3 \times 10^{5}$ cells/well. When the cells were $80 \%$ confluent, the recombined pGEM-T-GSN plasmids $(1 \mu \mathrm{l})$ were transfected into the four CC cell lines and the CCD-18Co normal cells using 3-5 $\mu$ 1 Lipofectamine ${ }^{\circledR} 2000$ per 200,000-1,000,000 cells (Thermo Fisher Scientific, Inc.).

Invasion and metastasis assays. Tumor cell invasion and metastasis were assessed using a Transwell insert (7 $\mu \mathrm{m}$; Corning, Inc., Corning, NY, USA). The SW480 and HT29 cells were grown to $85 \%$ confluence and then transfected with pGEM-T-GSN or the empty vector. Following transfection for $24 \mathrm{~h}$, the cells $\left(5 \times 10^{4}\right)$ were harvested, washed with phosphate-buffered saline, resuspended in $200 \mu \mathrm{l}$ serum-free medium and seeded into the upper chamber of the Transwell insert. A total of $600 \mu \mathrm{l}$ DMEM, containing $10 \% \mathrm{FBS}$ as a chemoattractant, was added to the lower chamber. For the invasion assay, the inserts were precoated with $30 \mu \mathrm{l}$ Corning Matrigel Matrix (Corning Inc.) and 6x10 4 cells were added to the upper chamber. Following incubation for $24 \mathrm{~h}$ at $37^{\circ} \mathrm{C}$ in a humidified atmosphere of $5 \% \mathrm{CO}_{2}$, non-migrating (non-invading) cells were removed from the upper surface of the filter with a cotton-tipped swab. The cells on the lower surface of the filter were fixed in $4 \%$ formaldehyde (Sigma-Aldrich) and stained with crystal violet staining solution (Sigma-Aldrich). Following staining, five randomly-selected fields were counted at a magnification of x100 using Eclipse 
E200-LED microscope (Nikon Corporation, Tokyo, Japan). All obtained data were from a minimum of three independent experiments performed in duplicate.

Western blot analysis. The cells and tissues were homogenized in radioimmunoprecipitation assay buffer, containing $20 \mathrm{mM}$ Tris- $\mathrm{HCl}$ (pH 7.5), $150 \mathrm{mM} \mathrm{NaCl}, 1 \mathrm{mM} \mathrm{Na}_{2}$ EDTA, $1 \mathrm{mM}$ EGTA, 1\% NP-40, 1\% sodium deoxycholate, $2.5 \mathrm{mM}$ sodium pyrophosphate, $1 \mathrm{mM} \beta$-glycerophosphate, $1 \mathrm{mM}$ $\mathrm{Na}_{3} \mathrm{VO}_{4}$ and $1 \mu \mathrm{g} / \mathrm{ml}$ leupeptin (Cell Signaling Technology, Inc., Danvers, MA USA). The protein concentration was determined using a Bicinchoninic Acid Protein Assay kit (Pierce Biotechnology, Inc., Rockford, IL, USA). Protein (20 $\mu \mathrm{g})$ was subjected to a 4-12\% gradient Bis-Tris Gel (Invitrogen; Thermo Fisher Scientific, Inc.) and electrotransferred onto a Hybond-enhanced chemiluminescence nitrocellulose membrane (GE Healthcare Life Sciences, Chalfont, UK). Following the transfer, the membrane was washed with $25 \mathrm{ml}$ Tris-buffered saline (TBS; Cell Signaling Technology, Inc.) for $5 \mathrm{~min}$ at room temperature, incubated in $25 \mathrm{ml}$ blocking buffer (Cell Signaling Technology, Inc.) for $1 \mathrm{hr}$ at room temperature, washed three times for $5 \mathrm{~min}$ each with $15 \mathrm{ml}$ TBS Tween 20 (TBST; Cell Signaling Technology, Inc.). The membrane was then incubated with rabbit anti-GSN monoclonal antibody (1:25,000, cat. no. ab7583; Abcam, Cambridge, MA, USA) overnight at $4^{\circ} \mathrm{C}$, washed three times for 5 min with $15 \mathrm{ml}$ TBST, and incubated with the goat anti-rabbit horseradish peroxidase-conjugated secondary antibodies (1:500) for $1 \mathrm{~h}$ at room temperature. Finally, it was washed three times for 5 min with $15 \mathrm{ml}$ TBST. The reaction was detected using an enhanced chemiluminescence system (GE Healthcare Life Sciences). The membranes were then reprobed using a mouse monoclonal anti-GAPDH antibody (cat. no. ab9484; Abcam) as an internal control.

Immunohistochemistry. Immunohistochemical analysis was performed on paraffin-embedded sections using an Envision kit (Dako, Glostrup, Denmark), according to the manufacturer's protocol. The sections were autoclaved for $10 \mathrm{~min}$ at $121^{\circ} \mathrm{C}$ for antigen retrieval. Anti-GSN monoclonal antibodies (Upstate Biotechnology Inc.) were applied to the sections at 1:100. The presence of staining was evaluated by a single pathologist, according to the overall level of the immunostaining.

Statistical analysis. Statistical analysis was performed using SPSS software, version 16.0 (SPSS, Inc., Chicago, IL, USA). The differences between variables were assessed by the $\chi^{2}$ test or Fisher's exact test. The survival rates of patients with CC were analyzed using Kaplan-Meier analysis, and a log rank test was used to compare the survival curves. Data derived from the cell line experiments are presented as the mean \pm standard deviation and assessed using a two-tailed Student's t-test. $\mathrm{P}<0.05$ was considered to indicate a statistically significant difference.

\section{Results}

Expression of GSN in the human CC specimens. The expression of GSN was detected in human CC specimens and CC cell lines (Fig. 1). A total of 30 paired primary colon tumor and corresponding normal colon tissue samples were screened using immunohistochemical staining with anti-GSN antibodies (Fig. 1A; right panel) and RT-qPCR. Partial sections from the same tissues were then stained with HE to confirm the presence of tumorous tissue (Fig. 1A; left panel). Tissues containing $>90 \%$ tumor cells were defined as tumors for further quantitative assessment of the expression of GSN using RT-qPCR.

Low expression levels of GSN are detected in CC cell lines. The expression levels of GSN were measured using RT-qPCR in the SW480, HT29, SW620 and HCT116 cell lines, and 30 paired CC and adjacent non-neoplastic colon tissues. The results demonstrated that the expression levels of GSN in the four CC cell lines were significantly lower, compared with those in the normal CCD-18Co cell line, with the lowest level in the SW480 cell line (Fig. 1B).

Expression of GSN is downregulated in the majority of CC specimens. The expression levels of GSN was high in the non-cancerous tissues. The mean expression levels of GSN in the $\mathrm{CC}$ tissue were significantly reduced, compared with the non-cancerous tissue $(\mathrm{P}<0.01$; Fig. $2 \mathrm{~A})$. Western blot analysis was performed to measure the protein expression levels of GSN in the four CC cell lines, and was compared with the results of the RT-qPCR. It was observed that the mRNA expression of GSN was reduced, with the greatest reduction in the SW480 cells (Fig. 1B). This was reflected by the western blotting, which indicated the greatest reduction in protein expression levels of GSN in the SW480 cells (Fig. 1C).

Low expression levels of GSN are associated with a poor metastasis-free survival (MFS) rate. To investigate the correlation between the clinicopathological parameters and the expression of GSN in patients with $\mathrm{CC}$, expression levels of GSN in the $30 \mathrm{CC}$ tissue specimens were measured using RT-qPCR. Low or high levels of GSN in the tumor were defined when the normalized expression of GSN resided in the $<50 \%$ or $>50 \%$ of the tumor, respectively. Accordingly, a low level of GSN was detected in 16/30 CC specimens (53.3\%), whereas a high level of GSN was detected in the remaining 14/30 CC specimens (46.6\%; Fig. 2C). Correlation analysis indicated that low expression levels of GSN were significantly associated with tumor metastasis ( $\mathrm{P}=0.012$; Fig. 2B). Kaplan-Meier analysis indicated that a low expression levels of GSN were associated with reduced MFS in the patients with CC $(\mathrm{P}=0.0428$; Fig. 2C).

High expression levels of GSN suppress the invasion of SW480 and HT29 cells in vitro. Overexpression of GSN in GSN transfected SW480 and HT29 cells, and vector-transfected SW480 and HT29 cells, measured using RT-qPCR (Fig. 3A) and western blotting (Fig. 3B). The expression of GSN in GSN-transfected SW480 and HT29 cells was higher compared with that in the control group $(\mathrm{P}<0.05)$. It was observed that the proliferation of SW480 and HT29 cells remained almost the same initially (Fig. 3C and D). Following transfection for $24 \mathrm{~h}$ with p-GEM-T-GSN or vector controls, vector-SW480 and vector-HT29 cells proliferated at a higher rate, compared with the GSN-transfected SW480 and HT29 
A

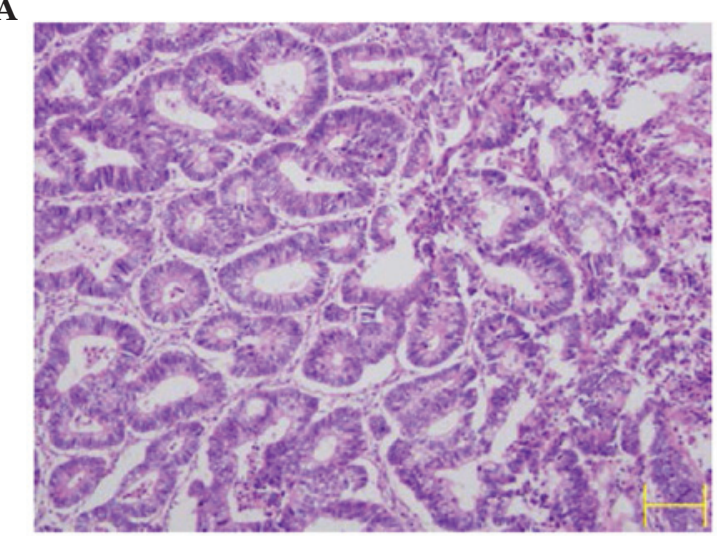

B
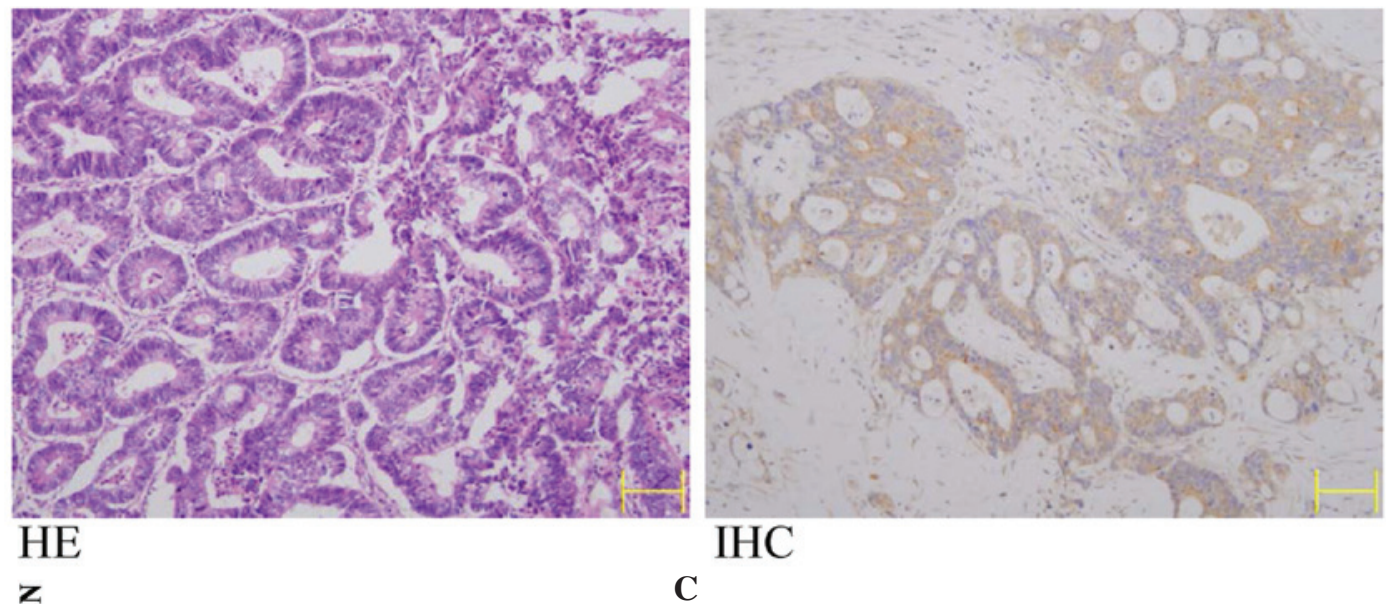

$\mathrm{IHC}$

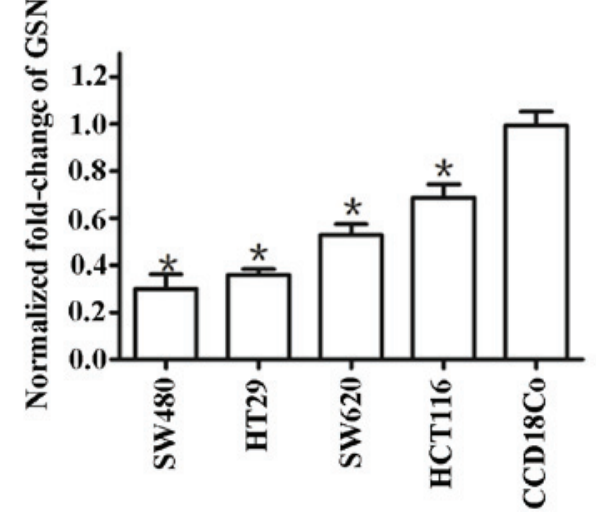

C
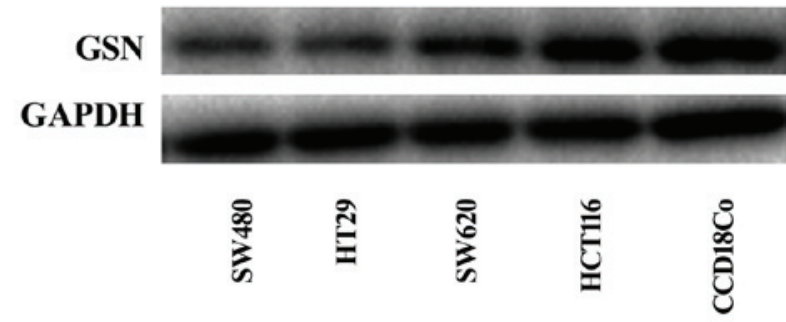

Figure 1. (A) Hematoxylin and eosin staining of carcinoma tissue (left panel) and immunohistochemical staining of carcinoma tissue with anti-GSN antibodies (right panel). Scale bar=50 mm. (B) mRNA expression levels of GSN in the colon carcinoma cell lines and normal CCD-18Co cells were measured using reverse transcription-quantitative polymerase chain reaction. " $\mathrm{P}<0.05$ compared with the CCD18Co group. Data are expressed as the mean \pm standard deviation. (C) Western blot analysis of the expression of GSN in the four colon carcinoma cell lines and normal CCD-18Co line. Magnification, x40. GSN, gelsolin; GAPDH, glyceraldehyde 3-phosphate dehydrogenase.

A

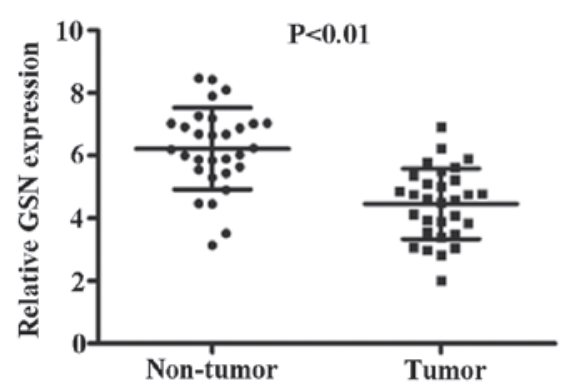

B

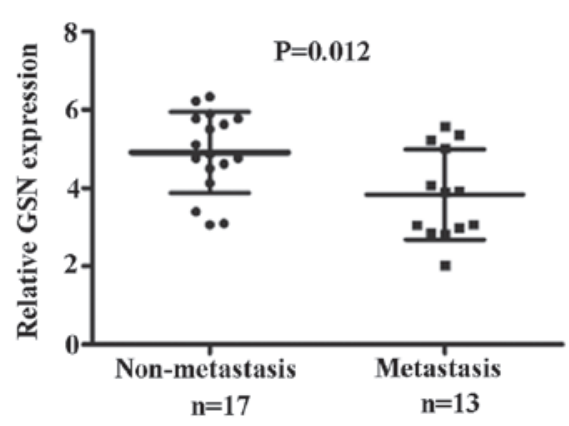

C

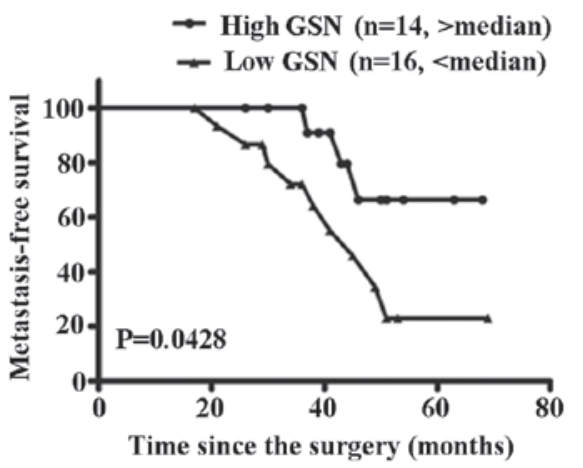

Figure 2. (A) Expression levels of GSN in 30 paired colon carcinoma and adjacent non-tumor tissues. Alterations in expression are presented as mean \pm standard deviation. The mean expression level of GSN in colon carcinoma was significantly reduced, compared with the non-tumor tissue. (B) Comparison of expression levels of GSN between colon carcinoma tissues with and without distant metastasis. The mean expression level of GSN in the distant metastasis group were significantly reduced, compared with those in the group without distant metastasis. (C) Kaplan-Meier analysis of the survival rates of patients with colon carcinoma as a function of GSN level. GSN, gelsolin.

cells. At 3 days post-transfection, the rate of proliferation was significantly different between the vector-transfected SW480 and HT-29 cells and their corresponding GSN-transfected cells $(\mathrm{P}=0.0312$ and $\mathrm{P}=0.0217$, respectively; Fig. $3 \mathrm{C}$ and $\mathrm{D})$, suggesting that the overexpression of GSN inhibited the proliferation of SW480 and HT29 cells.

Furthermore, the relative number of invasive cells in the vector-transfected cells was significantly lower, compared with the GSN-transfected SW480 cells ( $\mathrm{P}=0.023)$ and HT29 cells $(\mathrm{P}=0.011$; Fig. $3 \mathrm{E})$, suggesting that increased expression levels of GSN suppressed the invasion of the GSN-transfected SW480 and HT29 cells.

Overexpression of GSN reduces the expression levels of matrix metalloproteinase 2 (MMP2), BCL-2 and phosphorylated (p)-STAT3 in SW48O and HT29 cells. As presented in 
A

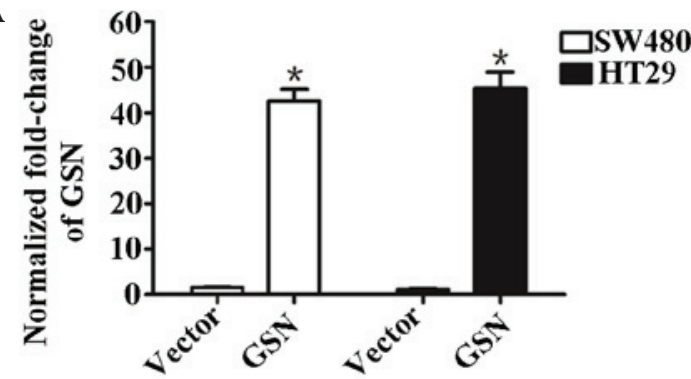

C

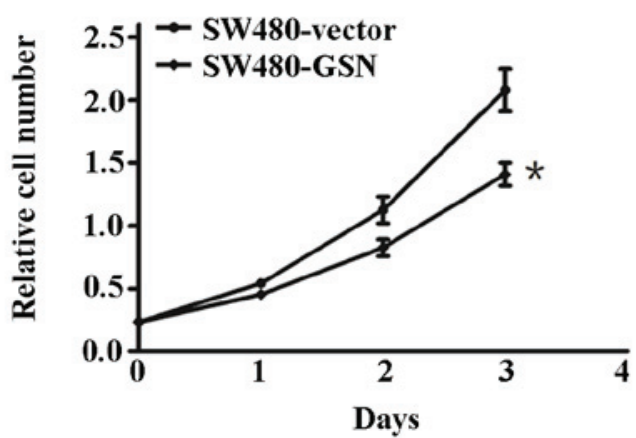

B

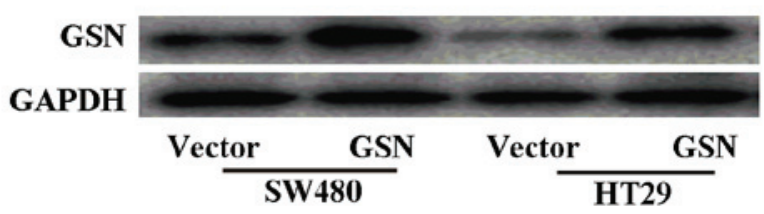

D
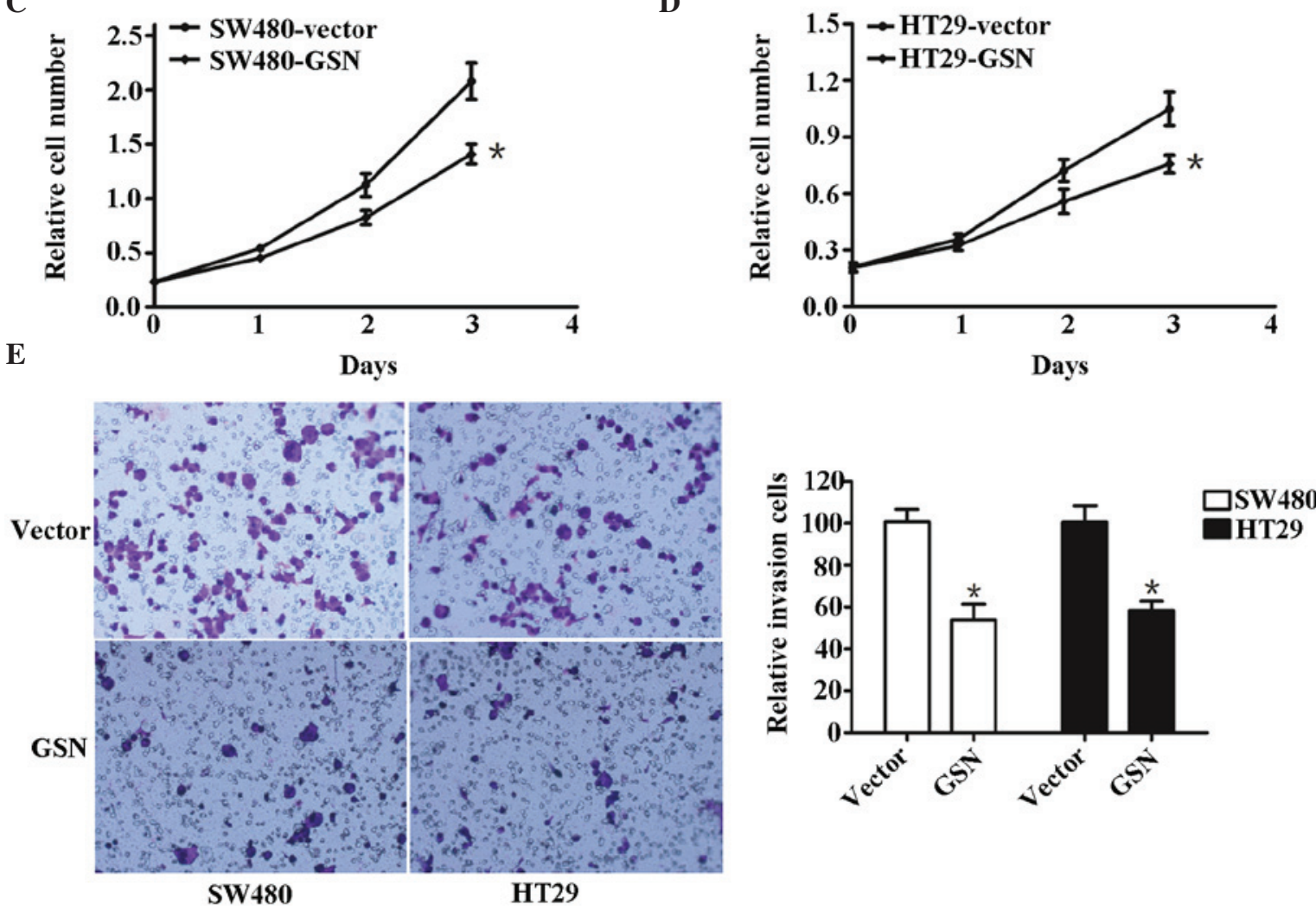

Figure 3. Overexpression of GSN in GSN-transfected SW480 and HT29 cells, and vector-transfected SW480 and HT29 cells, measured using (A) reverse transcription quantitative-polymerase chain reaction and (B) western blotting. Overexpression of GSN in GSN-transfected SW480 and HT29 cells was significantly higher, compared with the vector-transfected cells. Proliferation of the (C) vector- and GSN-transfected SW480 cells and (D) vector- and GSN-transfected HT29 cells were measured by relative cell numbers. (E) Crystal violet staining to determine the invasion of vector-transfected and GSN-transfected SW480 cells, and vector and GSN-transfected HT29 cells (magnification, x100). Data are expressed as the mean \pm standard deviation ("P<0.05). GSN, gelsolin; GAPDH, glyceraldehyde 3-phosphate dehydrogenase.

Fig. 4, the relative protein expression levels of STAT3 were not significantly reduced in the GSN-transfected SW480 and HT29 cells, compared with the vector-transfected SW480 and HT29 cells. However, the expression levels of MMP2, BCL-2 and p-STAT3 in the GSN-transfected SW480 and HT29 cells were significantly reduced, compared with the vector-transfected SW480 and HT29 cells $(\mathrm{P}=0.0231, \mathrm{P}=0.0326$ and $\mathrm{P}=0.0176$, respectively; Fig. 4). This indicated that the increased expression of GSN reduced the expression levels of MMP2, BCL-2 and p-STAT3 in the SW480 and HT29 CC cells.

\section{Discussion}

The abnormal regulation of cell migration is the primary cause of several diseases, including the invasion and migration of tumor cells. The migration of tumor cells across tissue barriers requires the degradation of specific components of the extracellular matrix, which triggers alterations in the interaction between the actin cytoskeleton and extracellular matrix proteins $(37,38)$. This process is affected by multiple factors, is dependant on adhesion molecule receptors and is regulated by specific actin binding factors (39). The involvement of specific actin binding factors in the migration of tumor cells has received increased attention.

GSN is a protein that is widely expressed intracellularly, including in the cytoplasm and mitochondria, and extracellularly, including the plasma (40). GSN can inhibit apoptosis by stabilizing mitochondria (41). Previous studies have demonstrated that GSN is involved in the regulation of epithelial-mesenchymal cell transformation (12), and that the expression of GSN expression can inhibit the migration potential of several types of human cancer cells (42).

In the present study, RT-qPCR was used to analyze the mRNA expression levels of GSN in CC cells, and the results 

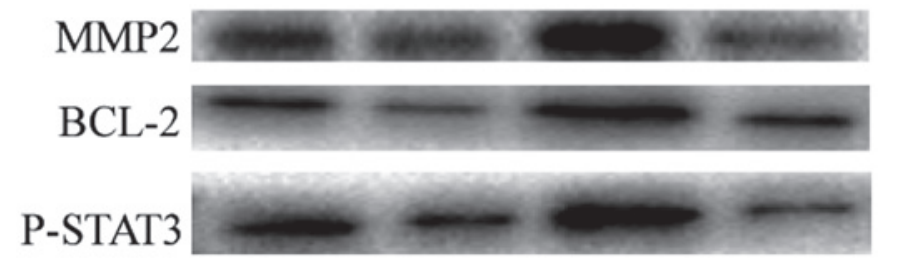

STAT3
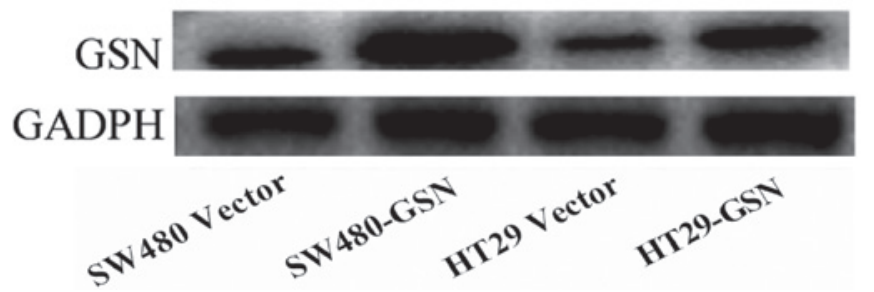

Figure 4. Western blot analysis of the expression levels of MMP2, BCL-2, STAT3 and P-STAT3 in vector- or GSN-transfected SW480 and HT29 cells. MMP-2, matrix metalloproteinase 2; STAT3, signal transducer and activator of transcription 3; P-, phosphorylated; GSN, gelsolin; GAPDH, glyceraldehyde 3-phosphate dehydrogenase.

revealed that there were significant reductions in the expression levels of GSN, compared with the levels of expression in the normal colon tissue (Fig. 1). In addition, the correlation between the expression levels of GSN and clinicopathological parameters was investigated, which indicated that the expression levels of GSN were reduced in 16/30 patients diagnosed with metastatic $\mathrm{CC}$, and were higher in the remaining 14 patients without metastatic CC. The mean expression level of GSN in the $\mathrm{CC}$ tissue was significantly reduced, compared with that in the non-cancerous tissue $(\mathrm{P}<0.01 ;$ Fig. $2 \mathrm{~A})$. Additionally, western blot analysis was performed in the present study to measure the expression levels of GSN in SW480, HT29, SW620 and HCT116 cell lines, which was observed to be downregulated in all four CC cell lines (Fig. 1C). These results suggested that GSN may be associated with the invasiveness of tumor cells, however, further investigations with a larger sample size are required to confirm this conclusion.

The results of the correlation analysis indicated that lower expression levels of GSN were associated with metastasis in the patients with $\mathrm{CC}(\mathrm{P}=0.012$; Fig. 2B). Kaplan-Meier analysis indicated that low expression levels of GSN were associated with the reduced rates of survival in patients with metastatic $\mathrm{CC}(\mathrm{P}=0.0428$; Fig. 2C). These results suggested that reduced expression of GSN may assist in the assessment of prognosis in patients with $\mathrm{CC}$, and may represent a novel prognostic marker in $\mathrm{CC}$.

A previous study reported the persistent activation of STAT proteins in several human cancer cell lines, including leukemia, multiple myeloma, breast cancer and prostate cancer (43). Cross-Knorr et al (44) reported that the low expression levels of P-STAT3, MMP-2 and BCL-2 were associated with the invasiveness of tumor cells. Notably, the present study indicated that the overexpression of GSN in SW480 and HT29 cell lines downregulated the expression levels of P-STAT3, MMP-2 and BCL-2. In addition, it was observed that the invasiveness of the SW480 and HT29 cells was reduced when GSN was overexpressed (Fig. 3). Therefore, the present study hypothesized that a low expression level of GSN results in persistent activation of P-STAT3, MMP-2 and BCL-2 via a specific molecular mechanisms, which further induces $\mathrm{CC}$ metastasis. By contrast, the overexpression of GSN may reduce this activation, thereby inhibiting $\mathrm{CC}$ metastasis.

Low or depleted expression levels of GSN appear to reflect the degree of CC malignancy, however, further investigations are required to validate the predictive value of GSN on the prognosis of CC.

In conclusion, to the best of our knowledge, the present study is the first to report the association between the mRNA expression of GSN and the survival rate of patients with metastatic CC. In addition, the present study indicated the potential diagnostic value of low expression levels of GSN as a prognostic factor in postoperative patients with $\mathrm{CC}$. The findings indicate the potential of using the mRNA expression level of GSN as a biomarker to assess the degree of tumor malignancy. However, whether GSN may be used as a treatment target or a marker of clinical tumor treatment requires further investigation.

\section{References}

1. Landherr L and Nagykálnai T: Chemotherapy of elderly patients with colorectal cancer. Magy Onkol 53: 97-105, 2009 (In Hungarian).

2. Meyerhardt JA and Mayer RJ: Systemic therapy for colorectal cancer. N Engl J Med 352: 476-487, 2005.

3. Jemal A, Murray T, Ward E, Samuels A, Tiwari RC, Ghafoor A, Feuer EJ and Thun MJ: Cancer statistics, 2005. CA Cancer J Clin 55: 10-30, 2005.

4. Brenner H, Kloor M and Pox CP: Colorectal cancer. Lancet 383: 1490-1502, 2014

5. Ohman U: Prognosis in patients with obstructing colorectal carcinoma. Am J Surg 143: 742-747, 1982.

6. Moreaux J and Catala M: Carcinoma of the colon: Long-term survival and prognosis after surgical treatment in a series of 798 patients. World J Surg 11: 804-809, 1987.

7. Slaby O, Svoboda M, Fabian P, Smerdova T, Knoflickova D, Bednarikova M, Nenutil R and Vyzula R: Altered expression of miR-21, miR-31, miR-143 and miR-145 is related to clinicopathologic features of colorectal cancer. Oncology 72: 397-402, 2007.

8. Addeo R, Montella L, Baldi A, Cennamo G, Guarrasi R, Faiola V, Caraglia M and Del Prete S: Atypical cutaneous lymphoid hyperplasia induced by chemotherapy in a patient with advanced colon carcinoma. Clin Colorectal Cancer 6: 728-730, 2007.

9. Dimitroff CJ, Sharma A and Bernacki RJ: Cancer metastasis: A search for therapeutic inhibition. Cancer Invest 16: 279-290, 1998.

10. Fidler IJ: Critical determinants of cancer metastasis: Rationale for therapy. Cancer Chemother Pharmacol 43 (Suppl): S3-S10, 1999.

11. Yamaguchi $\mathrm{H}$ and Condeelis $\mathrm{J}$ : Regulation of the actin cytoskeleton in cancer cell migration and invasion. Biochim Biophys Acta 1773: 642-652, 2007.

12. Yin HL and Stossel TP: Control of cytoplasmic actin gel-sol transformation by gelsolin, a calcium-dependent regulatory protein. Nature 281: 583-586, 1979.

13. Sun HQ, Yamamoto M, Mejillano M and Yin HL: Gelsolin, a multifunctional actin regulatory protein. J Biol Chem 274: 33179-33182, 1999.

14. Kwiatkowski DJ: Functions of gelsolin: Motility, signaling, apoptosis, cancer. Curr Opin Cell Biol 11: 103-108, 1999.

15. Tanaka H, Shirkoohi R, Nakagawa K, Qiao H, Fujita H, Okada F, Hamada J, Kuzumaki S, Takimoto M and Kuzumaki N: siRNA gelsolin knockdown induces epithelial-mesenchymal transition with a cadherin switch in human mammary epithelial cells. Int J Cancer 118: 1680-1691, 2006.

16. Thor AD, Edgerton SM, Liu S, Moore DH 2nd and Kwiatkowski DJ: Gelsolin as a negative prognostic factor and effector of motility in erbB-2-positive epidermal growth factor receptor-positive breast cancers. Clin Cancer Res 7: 2415-2424, 2001. 
17. Rao J, Seligson D, Visapaa H, Horvath S, Eeva M, Michel K, Pantuck A, Belldegrun A and Palotie A: Tissue microarray analysis of cytoskeletal actin-associated biomarkers gelsolin and E-cadherin in urothelial carcinoma. Cancer 95: 1247-1257, 2002.

18. Dosaka-Akita H, Hommura F, Fujita H, Kinoshita I, Nishi M, Morikawa T, Katoh H, Kawakami Y and Kuzumaki N: Frequent loss of gelsolin expression in non-small cell lung cancers of heavy smokers. Cancer Res 58: 322-327, 1998.

19. Chen P, Murphy-Ullrich JE and Wells A: A role for gelsolin in actuating epidermal growth factor receptor-mediated cell motility. J Cell Biol 134: 689-698, 1996.

20. Lader AS, Lee JJ, Cicchetti G and Kwiatkowski DJ: Mechanisms of gelsolin-dependent and -independent EGF-stimulated cell motility in a human lung epithelial cell line. Exp Cell Res 307: $153-163,2005$

21. Fujita H, Okada F, Hamada J, Hosokawa M, Moriuchi T, Koya RC and Kuzumaki N.: Gelsolin functions as a metastasis suppressor in B16-BL6 mouse melanoma cells and requirement of the carboxyl-terminus for its effect. Int J Cancer 93: 773-780, 2001.

22. Gao SP and Bromberg JF: Touched and moved by STAT3. Sci STKE 2006: pe30, 2006

23. Costantino L and Barlocco D: STAT 3 as a target for cancer drug discovery. Curr Med Chem 15: 834-843, 2008.

24. Aggarwal BB, Kunnumakkara AB, Harikumar KB, Gupta SR, Tharakan ST, Koca C, Dey S and Sung B: Signal transducer and activator of transcription-3, inflammation, and cancer: How intimate is the relationship? Ann N Y Acad Sci 1171: 59-76, 2009.

25. Aggarwal BB, Sethi G, Ahn KS, Sandur SK, Pandey MK, Kunnumakkara AB, Sung B and Ichikawa H: Targeting signal -transducer-and-activator-of-transcription-3 for prevention and therapy of cancer: Modern target but ancient solution. Ann N Y Acad Sci 1091: 151-169, 2006.

26. Berry DC, Levi L and Noy N: Holo-retinol-binding protein and its receptor STRA6 drive oncogenic transformation. Cancer Res 74: 6341-6351, 2014.

27. Ung N, Putoczki TL, Stylli SS, Ng I, Mariadason JM, Chan TA, Zhu HJ and Luwor RB: Anti-EGFR therapeutic efficacy correlates directly with inhibition of STAT3 activity. Cancer Biol Ther 15: 623-632, 2014.

28. Shen A, Chen Y, Hong F, Lin J, Wei L, Hong Z, Sferra TJ and Peng J: Pien Tze Huang suppresses IL-6-inducible STAT3 activation in human colon carcinoma cells through induction of SOCS3. Oncol Rep 28: 2125-2130, 2012.

29. Geinguenaud F, Souissi I, Fagard R, Motte L and Lalatonne Y: Electrostatic assembly of a DNA superparamagnetic nano-too for simultaneous intracellular delivery and in situ monitoring. Nanomedicine (Lond) 8: 1106-1115, 2012.

30. Lerner I, Hermano E, Zcharia E, Rodkin D, Bulvik R, Doviner V, Rubinstein AM, Ishai-Michaeli R, Atzmon R, Sherman Y, et al Heparanase powers a chronic inflammatory circuit that promotes colitis-associated tumorigenesis in mice. J Clin Invest 121: 1709-1721, 2011

31. Zugowski C, Lieder F, Müller A, Gasch J, Corvinus FM, Moriggl R and Friedrich K: STAT3 controls matrix metalloproteinase-1 expression in colon carcinoma cells by both direct and AP-1-mediated interaction with the MMP-1 promoter. Biol Chem 392: 449-459, 2011.
32. Tsareva SA, Wagner S, Müller A, Corvinus F and Friedrich K: Cell-cell contacts induce STAT3 activity in colon carcinoma cells through an autocrine stimulation loop. J Cancer Res Clin Oncol 137: 857-863, 2011.

33. Fenton JI and Birmingham JM: Adipokine regulation of colon cancer: Adiponectin attenuates interleukin-6-induced colon carcinoma cell proliferation via STAT-3. Mol Carcinog 49: 700-709, 2010

34. Tadlaoui Hbibi A, Laguillier C, Souissi I, Lesage D, Le Coquil S, Cao A, Metelev V, Baran-Marszak F and Fagard R: Efficient killing of SW480 colon carcinoma cells by a signal transducer and activator of transcription (STAT) 3 hairpin decoy oligodeoxynucleotide--interference with interferon-gamma-STAT1-mediated killing. FEBS J 276: 2505-2515, 2009.

35. O'Connell JB, Maggard MA and Ko CY: Colon cancer survival rates with the new American Joint Committee on Cancer sixth edition staging. J Natl Cancer Inst 96: 1420-1425, 2004.

36. Livak KJ and Schmittgen TD: Analysis of relative gene expression data using real-time quantitative PCR and the 2(-Delta Delta C(T)) Method. Methods 25: 402-408, 2001

37. Marín O, Rubenstein JL: Cell migration in the forebrain: Annu Rev Neurosci 26: 441-483, 2003

38. Martin TA, Ye L, Sanders AJ, Lane J and Jiang WG: Cancer Invasion and Metastasis: Molecular and Cellular Perspective. In: Madame Curie Bioscience Database [Internet]. Landes Bioscience 2000, Austin. Available from: http://www.ncbi.nlm. nih.gov/books/NBK164700/.

39. Linder S: The matrix corroded: Podosomes and invadopodia in extracellular matrix degradation. Trends Cell Biol 17: 107-117, 2007.

40. Wen D, Corina K, Chow EP, Miller S, Janmey PA and Pepinsky RB: The plasma and cytoplasmic forms of human gelsolin differ in disulfide structure. Biochemistry 35: 9700-9709, 1996.

41. Koya RC, Fujita H, Shimizu S, Ohtsu M, Takimoto M, Tsujimoto Y and Kuzumaki N: Gelsolin inhibits apoptosis by blocking mitochondrial membrane potential loss and cytochrome c release. J Biol Chem 275: 15343-15349, 2000.

42. Tanaka M, Müllauer L, Ogiso Y, Fujita H, Moriya S, Furuuchi K, Harabayashi T, Shinohara N, Koyanagi T and Kuzumaki N: Gelsolin: A candidate for suppressor of human bladder cancer. Cancer Res 55: 3228-3232, 1995.

43. Buettner R, Mora LB and Jove R: Activated STAT signaling in human tumors provides novel molecular targets for therapeutic intervention. Clin Cancer Res 8: 945-954, 2002.

44. Cross-Knorr S, Lu S, Perez K, Guevara S, Brilliant K, Pisano C, Quesenberry PJ, Resnick MB and Chatterjee D: RKIP phosphorylation and STAT3 activation is inhibited by oxaliplatin and camptothecin and are associated with poor prognosis in stage II colon cancer patients. BMC Cancer 13: 463, 2013. 